\title{
Analog VLSI System for Active Drag Reduction
}

\author{
Bhusan Gupta, Rodney Goodman, Fukang Jiang, Yu-Chong Tai \\ Dept. of Electrical Engineering \\ California Institute of Technology \\ Pasadena, CA 91125 \\ Steve Tung, Chih-Ming Ho \\ MANE \\ UCLA \\ Los Angeles CA 90024 \\ E-mail: bgupta@caltech.edu
}

\begin{abstract}
We describe an analog CMOS VLSI system that can process real-time signals from surface-mounted shear stress sensors to detect regions of high shear stress along a surface in an airflow. The outputs of the CMOS circuit are used to actuate micromachined flaps with the goal of reducing this high shear stress on the surface and thereby lowering the total drag. We have designed, fabricated, and tested parts of this system in a wind tunnel in laminar and turbulent flow regimes.
\end{abstract}

\section{1: Introduction}

In today's cost-conscious air transportation industry, fuel costs are a substantial economic concern. Drag reduction is an important way to increase fuel efficiency which reduces these costs. It is estimated that even a $5 \%$ reduction in drag can easily translate into savings of millions of dollars in annual fuel costs.

Counter-rotating vortices

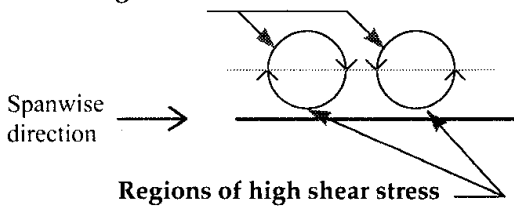

Flow into page

Figure 1 Diagram of the interaction of a vortex pair and the wall.

Large skin friction drag has recently been linked to organized structures in turbulent airflows which play an important role in turbulence transport. The main cause of high drag in turbulent flows is commonly observed nearwall streamwise vortices (Figure 1). The interaction of These vortices, which appear randomly in both space and time, interact with the viscous layer near the walls creating high local regions of high surface shear stress which contributes to the total drag. Therefore, attempts to reduce drag by controlling turbulent flows have focused on methods of either preventing the formation or mitigating the strength of these vortices. The small size of these vortices, which decreases as the Reynolds number of the flow increases, has limited physical experimentation, and the inherent complexity of the non-linear governing Navier-Stokes equations has likewise limited the analytical approaches.

\section{2: Can biology tell us something?}

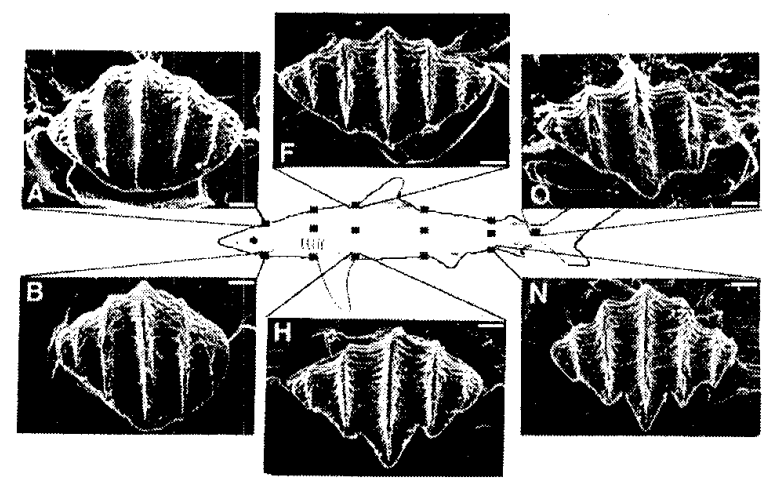

Figure 2 Examples of shark scales (white bar $=25 \mu \mathrm{m}$ ).

In many complex problems, one can often find inspiration by observing how nature has evolved 
biological systems to address the problem. For the problem of drag reduction, deep sea sharks serve as a possible biological model because they are a highly evolved predator with a 350 million year old lineage. Deep-sea sharks (e.g. hammerhead sharks) can swim up to $20 \mathrm{~m} / \mathrm{s}$ in deep water. Little is known about the physiology of these species because they are difficult to study as the deep sea setting is hard to replicate in a controlled environment. Biologists do know, however, something about the scales (dermal denticles) that cover the shark's skin. Only recently [1] the denticles have been found to have microscopic structure to them (Figure 2). The natural argument about evolution would lead one to conclude that the structure of these scales assists the sharks' movement perhaps indicating some method of underwater drag reduction.

\section{1: Active Control}

The question of active control in sharks is even more of a unknown quantity. It is theorized [2] that sharks can actively move their denticles. The indirect evidence of this is twofold: one, the denticles are connected to muscles underneath the shark's skin, and two, the number of mechano-receptive pressure sensors (pit organs) on a shark's body is positively correlated with the speed of the species. But whether sharks actually utilize active control remains an unanswered question. The conclusion that one can draw from this example of biology is that it may be beneficial to employ microscopic actively controlled structures to reduce the drag.

\section{3: How Small Is Small?}

By specifying the problem of drag over a flat surface at airflow velocities of 10 to $20 \mathrm{~m} / \mathrm{s}$, one can define statistics about the scope of the problem. The drag-inducing vortex pair streaks vary as the Reynolds number of the flow changes. For a typical airflow of $15 \mathrm{~m} / \mathrm{s}$ in the wind tunnel the Reynolds number is about $10^{4}$ which means the vortex streaks have a statistical mean width of about $1 \mathrm{~mm}$. The length of a typical vortex streak can be about $2 \mathrm{~cm}$ giving the streaks a $20: 1$ aspect ratio. The average spacing between streaks is about $2.5 \mathrm{~mm}$. The frequency of appearance of the streaks, is approximately $100 \mathrm{~Hz}$. The appearance and disappearance of these vortex pairs can be best be described by a chaotic process.

\section{1: Computer simulations}

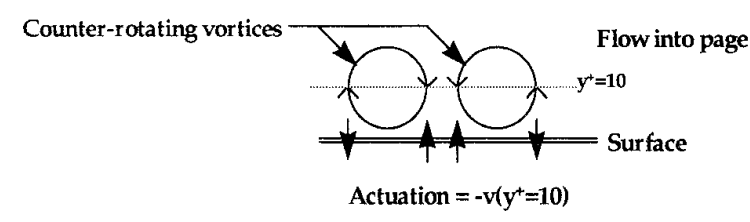

Figure 3 Simple control law for $\mathbf{2 5 \%}$ drag reduction.

Numerical experiments have demonstrated significant drag reduction $(\sim 25 \%)$ can be achieved by suppression of the interaction between stream-wise vortices and the wall through active feedback control [3]. The active control scheme used in the experiments involved blowing and suction at the wall according to the normal component of the velocity field $\left(-\mathrm{v}\left(\mathrm{y}^{+}=10\right)\right)$ sensed in the near-wall region away from the surface (see Figure 3 ). It appears that even a simple control rule that pushes the region of high shear stress away from the wall is beneficial.

\section{4: System Details}

The goal is to design a system that incorporates VLSI control circuitry along with microscopic sensors and actuators and control circuitry that can actively deform its surface to reduce drag.

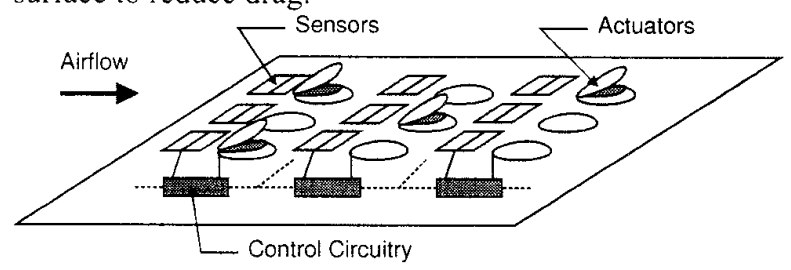

Figure 4 Simplified diagram of the hardware system.

Figure 4 shows the physical layout of our system - the surface is covered with both sensors and actuators controlled by circuitry underneath.

The signals from the sensors are processed to detect regions of high shear stress. To accomplish this detection, information about the physical and temporal nature of the vortex pair streaks is considered. First, the skewed aspect ratio (long and narrow) leads to building "column"oriented templates for streak detection. The sensor outputs are organized into thin strips oriented in the direction of the airflow. When several sensors in a column register either a larger or smaller output than its neighbors in a spanwise direction, this difference is accumulated. If the difference exceeds a threshold, it is claimed that a vortex pair streak is present in that column and an appropriate control action needs to be taken which is to energize the associated actuator. 


\section{1: Micromachined Components}

Silicon micromachining technology is utilized to build the microsensors and microactuators $[4,5,6]$.

\subsection{1: Shear Stress Sensor:}

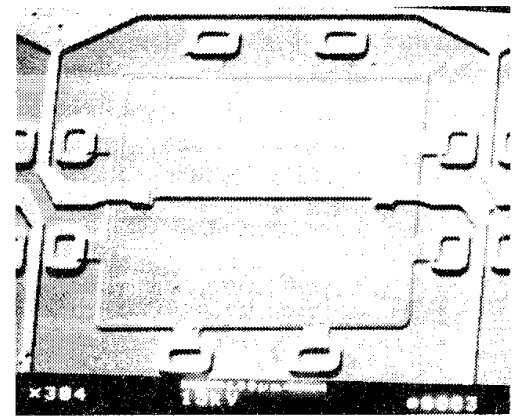

Figure 5 Shear stress sensor showing polysilicon wire over diaphragm.

The microsensor allows measurement of the heat transfer between a heated wire and the air. As the wire is heated electrically, heat is transferred by convection to the fluid flow causing a power change in the polysilicon wire. The polysılicon wire sits on a $200 \mu \mathrm{m}$ square, $1.2 \mu \mathrm{m}$ thick silicon nitride diaphragm over a vacuum cavity. The $2.0 \mu \mathrm{m}$ deep vacuum cavity serves to minimize thermal losses to the substrate.

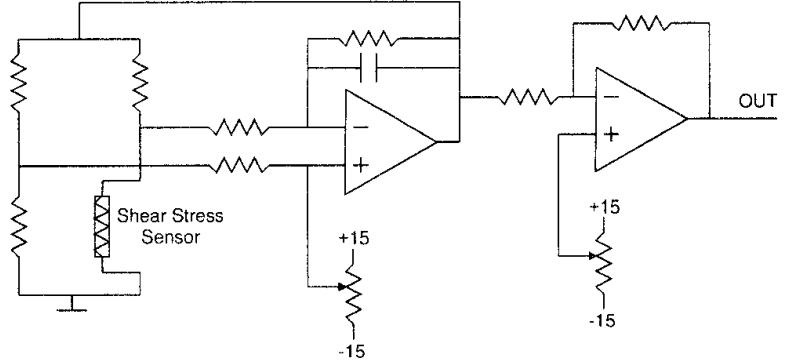

Figure 6 Constant Temperature Circuit for the Shear Stress Sensor

The shear stress sensor is controlled by a discrete constant temperature (CT) circuit. The circuit maintains a constant temperature on the heated wire by means of balancing a bridge (Figure 6). The amplified voltage feedback signal is used for the output. The overall gain of the CT circuit is designed to be about 20 .

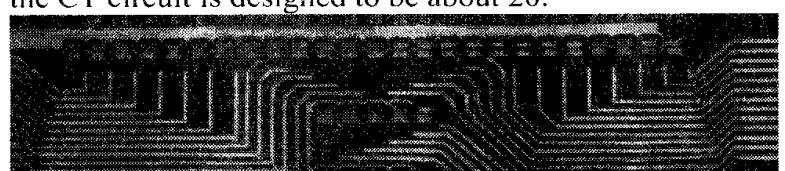

Figure 7 Array of shear stress sensors.

The sensors used in this experiment have been fabricated as a single row of 25 sensors (Figure 7). This allows monitoring of a spanwise section of the channel. Typically five of the middle sensors are used as inputs to the detection/control chip. Because we have many fewer actuators than sensors, it is unnecessary to use more sensors in the detection experiments.

\subsection{2: Microactuator:}

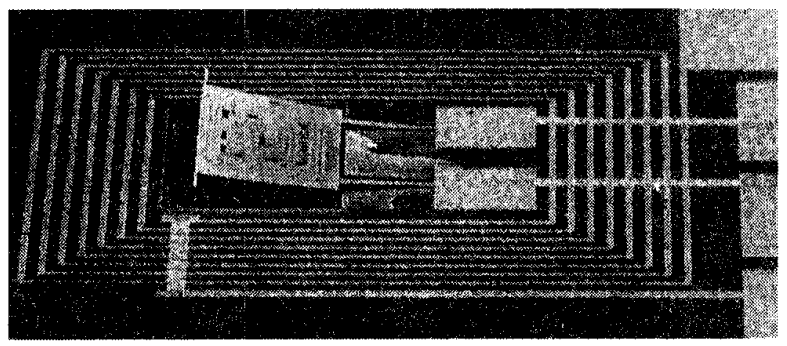

Figure 8 Photograph of a microactuator.

The microactuator [7] is a thin plate that is raised via magnetic actuation of a layer of permalloy. Current through a coil of metal is enough to cause up to $100 \mu \mathrm{m}$ deflections. The microactuators suffer from the lack of a high mechanical bandwidth. In theory, the resonant frequency is about $1 \mathrm{kHz}$. However, only a $100 \mathrm{~Hz}$ bandwidth is physically achievable currently.

\section{2: Circuits}

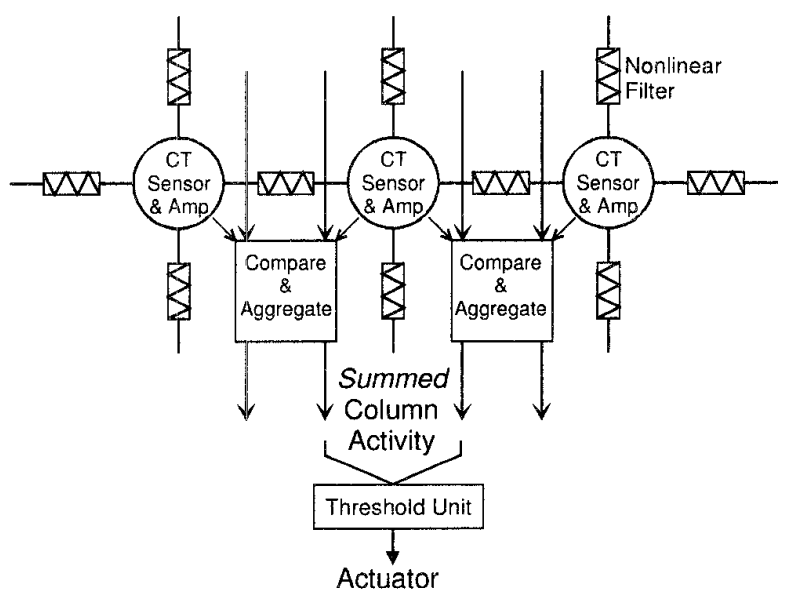

Figure 9 Block diagram of the complete detection/control chip.

Figure 9 shows a diagram of how the information is processed in the detection/control chip. A non-linear filtering network connects together the amplified outputs of the shear stress sensors. The filtering preserves large differences between adjacent sensors while smoothing small differences. The comparison and aggregation is 
organized by columns corresponding to different actuators. Once the signal is aggregated and exceeds a threshold then the actuator is driven.

A detailed schematic of a single column (or channel) is presented in Figure 10. The CT output sensor signal is fed into a further stage of amplification (Figure 11). The amplified signal is buffered (Figure 12) and distributed to a non-linear resistive network composed of the HRES circuits [8] (Figure 13). The spatial filtering constant is different between sensor outputs in the same column and sensor outputs in adjacent columns. The different biases serve to reinforce activity in a column and discourage activity in adjacent columns. The filtered signals are then fed to a symmetric anti-bump circuit [9] (Figure 14). The circuit's operation can be described as a soft comparator with an adjustable dead zone. The function of the circuit is to indicate when a particular column has registered a large shear stress value while the neighboring columns have not. The output of the anti-bump circuit, a current, is summed for a particular column and compared to a threshold using the circuit in Figure 15. The actuator is triggered if the threshold is exceeded.

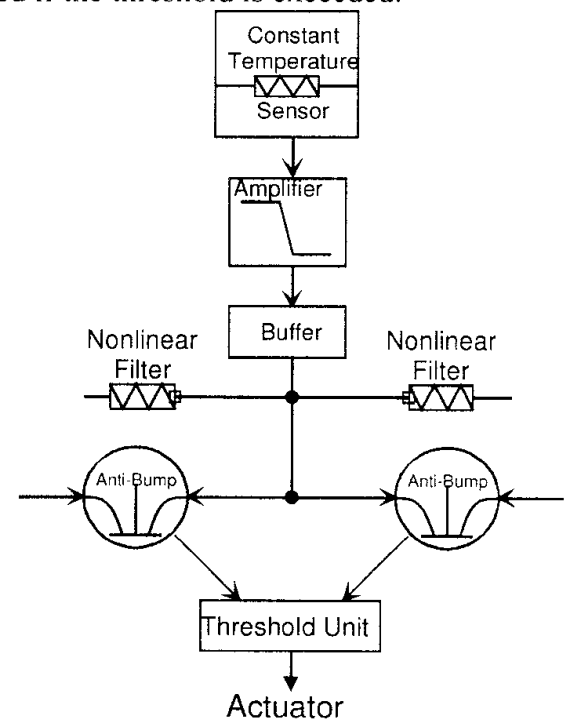

Figure 10 Schematic of one column (channel) of the detection/control circuitry

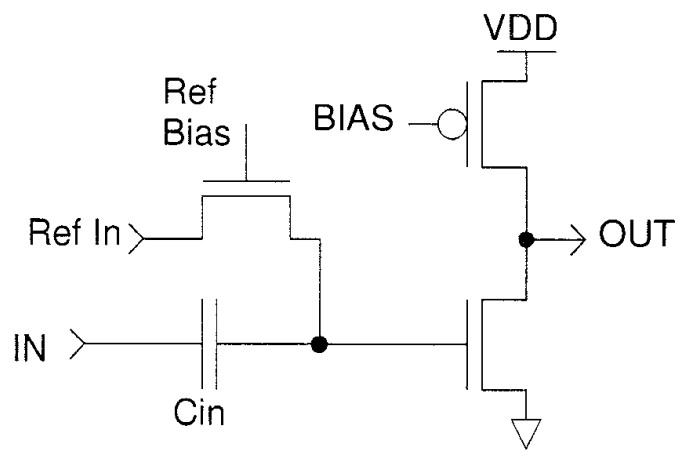

Figure 11 Schematic of the first stage amplifier.

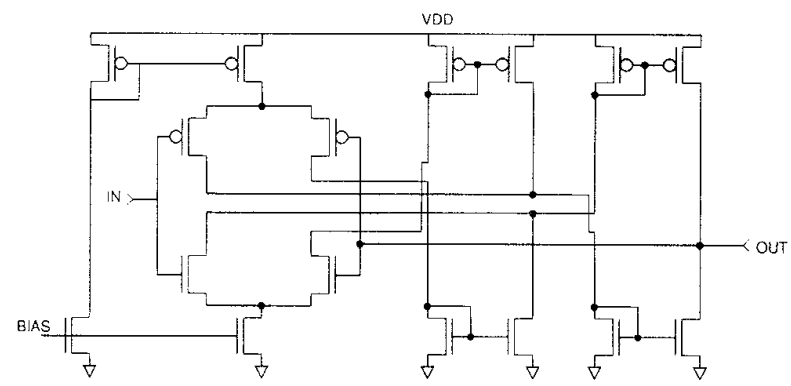

Figure 12 Schematic of a rail to rail buffer amplifier.

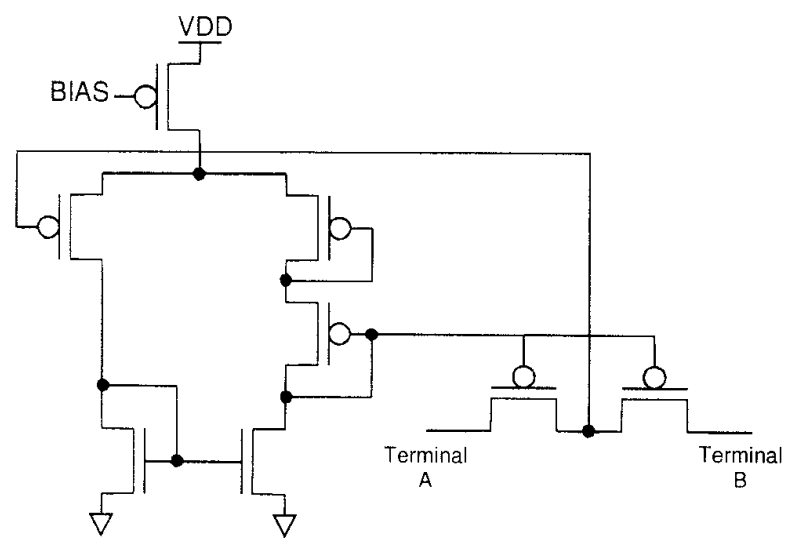

Figure 13 Schematic of the nonlinear resistor (HRES). 


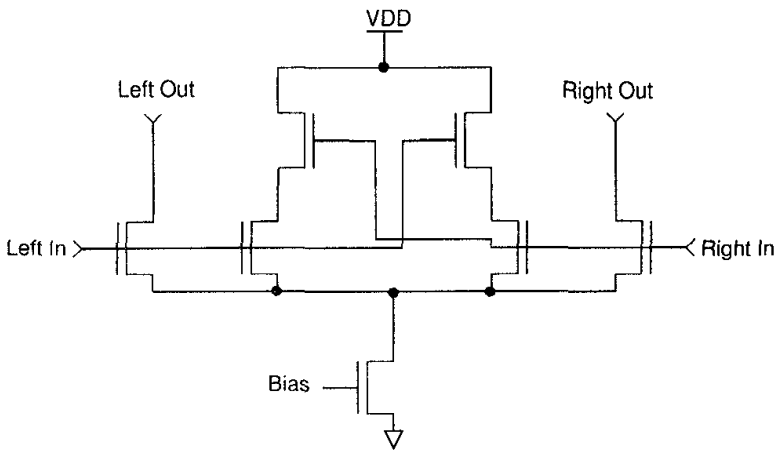

Figure 14 Schematic of the symmetric anti-bump circuit

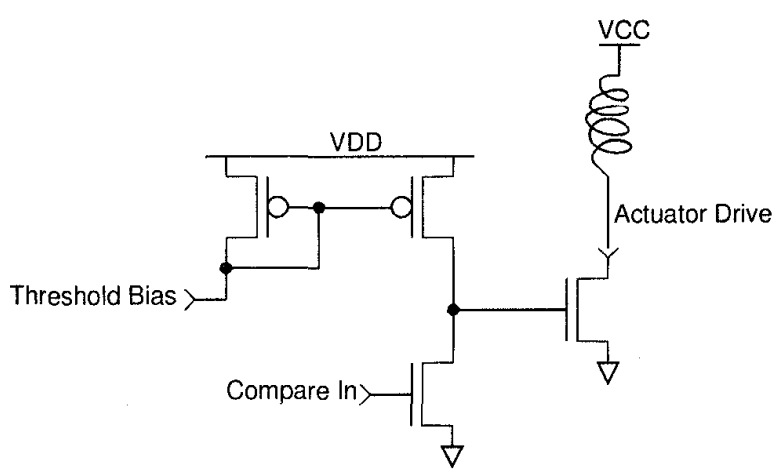

Figure 15 Schematic of the threshold comparator circuit.

The detection/control chip was fabricated using the $2.0 \mu \mathrm{m}$ CMOS process available through Mosis (i.e. Orbit Semiconductor N-Well). A chip plot is shown in Figure 16.

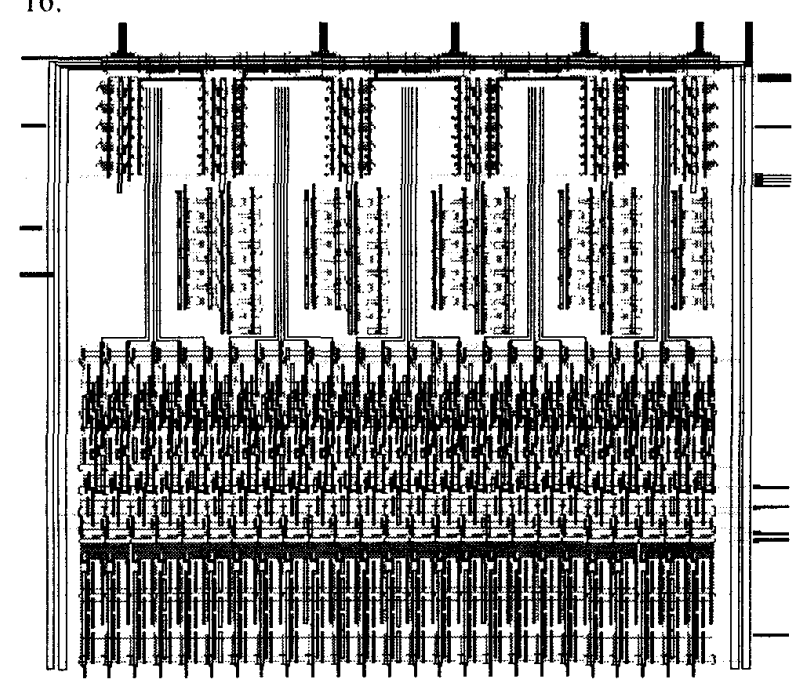

Figure 16 Plot of the detection/control chip.

\section{5: System Testing}

Several different experiments can test various components of the system. First, artificial signals are presented to the detection/control chip and the outputs verified. Second, in a laminar flow in the wind tunnel known disturbances are mechanically generated to experiment with the response of the detection/control chip and sensor/actuator system. Third, the fully turbulent channel flow is presented to the sensors and the response of the chip is observed.

\section{1: Lab bench results}

The overall delay of the processing system is about $40 \mu \mathrm{s}$. The amplifier in Figure 11 has a gain of about 10 and a bandwidth of about $50 \mathrm{kHz}$. The buffer has a full power bandwidth of about $100 \mathrm{kHz}$. The delay through the circuits in Figure 14 and Figure 15 accounts for the remainder of the time delay. The actuator driver can sink about $30 \mathrm{~mA}$.

\section{1: Sample test of system in laminar flow}

This experiment consisted of generating a periodic disturbance in a laminar flow in order to check the response of the signal processing and the actuator movement. The disturbance was created by a macro-sized actuator driven by an signal generator. The perturbation frequency is about seven hertz which is reasonable for the microactuator to follow.

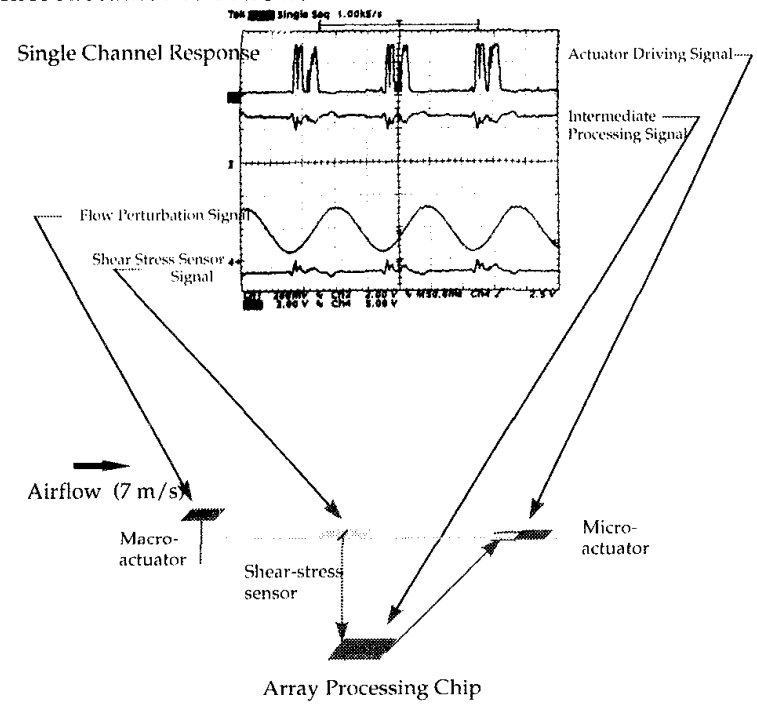

Figure 17 Laminar flow perturbation experiment. 


\section{2: Turbulent Drag Performance}

Reducing the fully turbulent drag is the experiment that this system is designed for. The system is presented with a fully turbulent airflow profile. The centerline velocity of the channel varies between $10 \mathrm{~m} / \mathrm{s}$ and $20 \mathrm{~m} / \mathrm{s}$. In this experiment, the microactuators do not yet have the mechanical frequency response to follow the actuation signals. Thus, only an rough estimate of the drag reduction that our system provides is possible. Both the outputs of the shear stress sensors and the outputs of the detection/control chip are recorded. The estimated drag reduction is approximately $2.39 \%$ assuming that the microactuators are about $75 \%$ effective in reducing the high shear stress.

The single column response of the system is graphed in Figure 18.
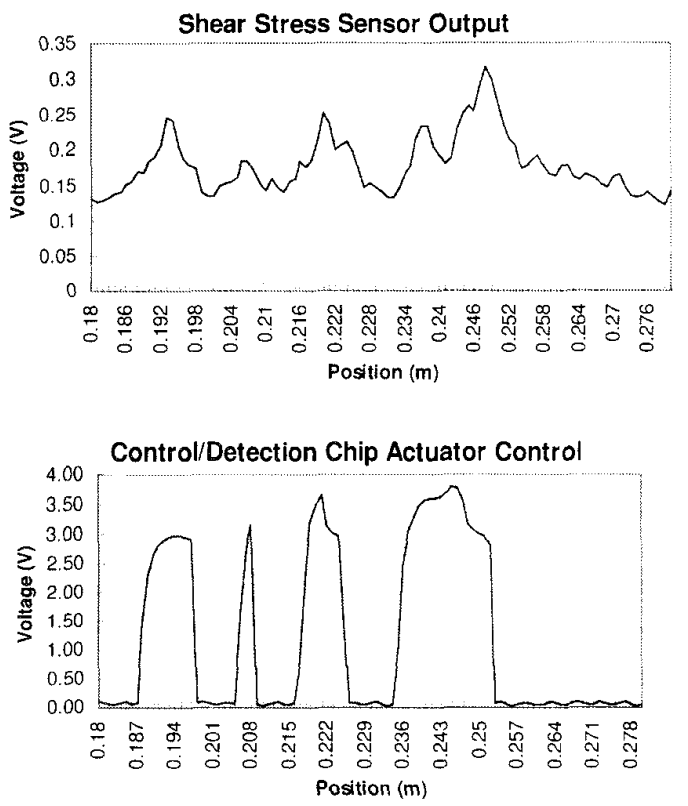

Figure 18 Single column waveforms of the shear stress sensors and detection/control chip in a turbulent flow.

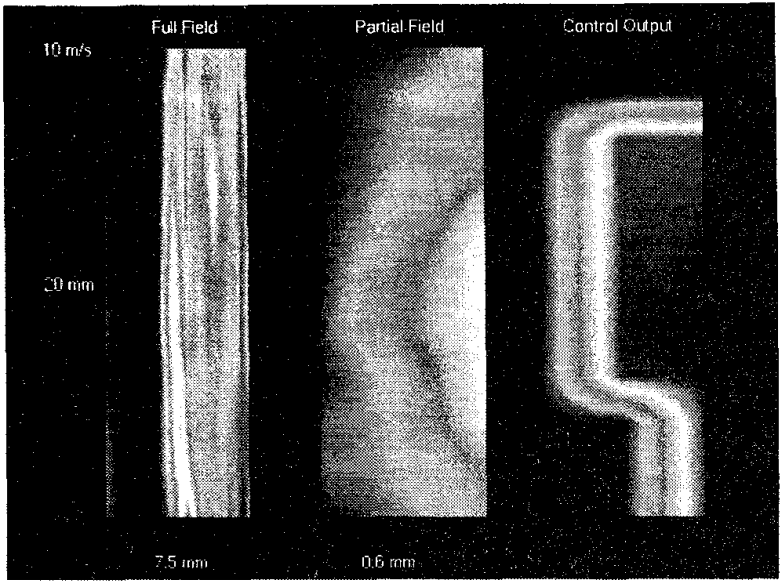

Figure 19 Flow snapshot showing sensor outputs as well as detection/control chip outputs at $10 \mathrm{~m} / \mathrm{s}$.

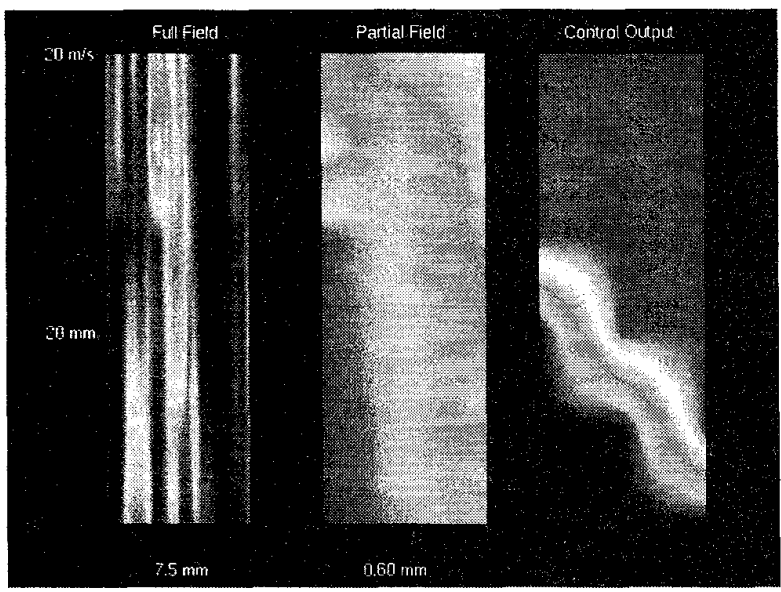

Figure 20 Flow snapshot showing sensor outputs as well as detection/control chip outputs at $20 \mathrm{~m} / \mathrm{s}$.

Figure 19 and Figure 20 both show a plot of the full field of 25 sensors and the reduced five sensor field used to generate the detection/control output.

\section{6: Conclusions}

We have described an analog VLSI system that can be used to actively reduce drag in an integrated system. We are in the process of verifying the actual drag reduction by controlling microactuators in wind tunnel experiments. We are encouraged that an approach similar to one biology may employ provides a very useful contribution to problem of drag reduction. 


\section{Acknowledgments:}

This work is supported in part by the Center for Neuromorphic Systems Engineering as a part of the National Science Foundation Engineering Research Center Program under grant EEC-9402726; and by the California Trade and Commerce Agency, Office of Strategic Teclnology under grant C94-0165. This work is also supported in part by ARPA/ONR under grant no. N00014-93-1-0990, and by an AFOSR University Research Initiative grant no. F49620-93-1-(0332.

\section{References}

[1] D.W. Bechert, G. Hoppe, W.-E. Reif, "On the Drag Reduction of the Shark Skin", AIAA paper No. 85-0546, 1985.

[2] W.-E. Reif and A. Dinkelacker, "Hydrodynamics of the squamation in fast-swimming sharks", Netues Jahrb. Geol. Paläontol. Ahh., (1982), vol. 164, pp. 184-187.

[3] P. Moin, J. Kim, H. Choi, "On Active Control of WallBounded Turbulent Flows," AIAA Paper No. 89-0960, 1989.

[4] P.R. Bandyopadhyah, "Development of a Microfabricated Surface for Turbulence Diagnostics and Control," ASME Application of Microfahrication to Fluid Mechanics, Chicago (1994). pp. 67-74.

[5] C. Liu, Y.C. Tai, J.B. Huang, C.M. Ho, "Surface Micromachined Thermal Shear Stress Sensor," ASME Application of Microfabrication to Fluid Mechanics, Chicago (1994), pp. 9-15.

[6] F. Jiang, Y.C. Tai, J.B. Huang, C.M. Ho, "Polysilicon Structures for Shear Stress Sensors," to be published in Tech. Digest IEEE TENCON'95, Hong Kong, Nov. 1995.

[7] T. Tsao, C. Liu, Y.C. Tai, C.M. Ho, Micromachined Magnetic Actuator for Active Fluid Control," $A S M E$ Application of Microfabrication to Fluid Mechanics, Chicago (1994), pp. 31-38.

[8] C.A. Mead, Analog VLSI and Neural Systems, AddisonWesley, Reading, Massachusetts (1989).

[9] T. Delbrück, "Bump Circuits for computing similarity and dissimilarity of analog voltages," Computation and Neural Svstems Dept. Memo 10, California Institute of Technology, Pasadena (1991). 